IFUP-TH $3 / 95$

\title{
Topological charge density renormalization in the presence of dynamical fermions.
}

\author{
B. Allés, A. Di Giacomo, H. Panagopoulos ${ }^{\star}$, E. Vicari \\ Dipartimento di Fisica dell'Università and I.N.F.N., I-56126 Pisa, Italy \\ * Department of Natural Sciences, University of Cyprus, Nicosia, Cyprus
}

\begin{abstract}
We study the renormalization group behaviour of the topological charge density in full QCD on the lattice. We propose a way of extracting the necessary renormalization functions from Monte Carlo simulations.
\end{abstract}

One important issue which has come up in recent years, in the study of strong interactions, is the so called proton spin crisis [1] 3]. The issue concerns the on-shell nucleon matrix element of the singlet axial current $j_{\mu}^{5}$

$$
\left\langle\vec{p}, e\left|j_{\mu}^{5}\right| \vec{p}^{\prime}, e^{\prime}\right\rangle=\bar{u}(\vec{p}, e)\left[G_{1}\left(k^{2}\right) \gamma_{\mu} \gamma_{5}-G_{2}\left(k^{2}\right) k_{\mu} \gamma_{5}\right] u\left(\vec{p}^{\prime}, e^{\prime}\right)
$$

where $e, e^{\prime}$ label the helicity states and $k$ is the momentum transfer. In a naive wave function picture $G_{1}(0)$ can be interpreted as the fraction of the nucleon spin carried by the quarks. Experimental determinations lead to an unexpectedly small value of $G_{1}(0)$, calling for a theoretical explanation in the context of a nonperturbative study in QCD. $G_{1}(0)$ can be extracted from the nucleon matrix element of the topological charge density $q(x)$ by using the fact that in the chiral limit the anomalous divergence of $j_{\mu}^{5}$ is proportional to the topological charge density. In the presence of dynamical fermions, one has

$$
\left\langle\vec{p}, e|q| \vec{p}^{\prime}, e^{\prime}\right\rangle=M B\left(k^{2}\right) \bar{u}(\vec{p}, e) i \gamma_{5} u\left(\vec{p}^{\prime}, e^{\prime}\right),
$$

with

$$
N_{f} B(0)=G_{1}(0) .
$$

Preliminary lattice studies of the on-shell matrix element of $q(x)$, in quenched QCD, have been done in Refs. [1 [6]. An unquenched study using a geometrical definition of topological charge density has been reported in Ref. [7]. We also would like to note the direct calculations of the on-shell proton $j_{\mu}^{5}$ matrix element performed in quenched QCD reported in Refs. [8.9].

Notice that the above discussion overlooks the non-invariance under the renormalization group of the operators involved; indeed, unlike pure gauge theory, in full QCD the topological 
charge density mixes under renormalization with $\partial_{\mu} j_{\mu}^{5}$. Therefore the relations of the matrix elements of $j_{\mu}^{5}$ and $q(x)$ with the experimental observables like $G_{1}(0)$ cannot be strictly given by Eqs. (1) and (3). Instead, one must look for equations involving renormalization group invariant quantities. This will appear clearer in the following, when we analyze the renormalization of operators regularized on the lattice.

In this letter we address the scale dependence of the matrix elements of operators entering the anomaly equation, in lattice regularization, and provide the prescriptions necessary to undertake a lattice calculation of $G_{1}(0)$ in full QCD.

Let us first discuss this issue in the case of dimensional regularization with minimal subtraction. In euclidean space

$$
\partial_{\mu} j_{\mu}^{5}(x)=i 2 N_{f} q(x)+i 2 p(x)
$$

where

$$
\begin{aligned}
j_{\mu}^{5}(x) & =\sum_{f=1}^{N_{f}} \bar{\psi}_{f}(x) \gamma_{\mu} \gamma_{5} \psi_{f}(x), \\
q(x) & =\frac{g^{2}}{64 \pi^{2}} F_{\mu \nu}^{a}(x) \tilde{F}_{\mu \nu}^{a}(x), \\
p(x) & =\sum_{f=1}^{N_{f}} m_{f} \bar{\psi}_{f}(x) \gamma_{5} \psi_{f}(x) .
\end{aligned}
$$

The nonrenormalizability property of the anomaly in the $\overline{\mathrm{MS}}$ scheme means that the anomaly equation should take exactly the same form in terms of bare or renormalized quantities. However the renormalization of the three pseudoscalar gauge invariant operators $\partial_{\mu} j_{\mu}^{5}(x)$, $q(x)$ and $p(x)$ turns out to be nontrivial; in the $\overline{\mathrm{MS}}$ scheme it reads 10,11]

$$
\left(\begin{array}{c}
i 2 N_{f} q(x) \\
\partial_{\mu} j_{\mu}^{5}(x) \\
i 2 p(x)
\end{array}\right)^{R}=\left(\begin{array}{ccc}
1 & z-1 & 0 \\
0 & z & 0 \\
0 & 0 & 1
\end{array}\right)\left(\begin{array}{c}
i 2 N_{f} q(x) \\
\partial_{\mu} j_{\mu}^{5}(x) \\
i 2 p(x)
\end{array}\right)^{B}
$$

where, to order $g^{4}$,

$$
z=1-\frac{g^{4}}{16 \pi^{4}} \frac{3 c_{F}}{8} N_{f} \frac{1}{\epsilon}+O\left(g^{6}\right)
$$

$\epsilon=d / 2-2$ and $c_{F}=\frac{N^{2}-1}{2 N}=\frac{4}{3}$. The structure of the renormalization matrix above assures the stability of the anomaly equation under renormalization. The associated anomalous dimension is given by

$$
\gamma_{\overline{\mathrm{MS}}}(g)=\mu \frac{\mathrm{d} Z}{\mathrm{~d} \mu} Z^{-1}=\left(\begin{array}{ccc}
0 & \bar{\gamma}(g) & 0 \\
0 & \bar{\gamma}(g) & 0 \\
0 & 0 & 0
\end{array}\right)
$$

where

$$
\bar{\gamma}(g)=\mu \frac{\mathrm{d}}{\mathrm{d} \mu} \ln z=\gamma_{2} g^{4}+\gamma_{3} g^{6}+O\left(g^{8}\right)
$$


and

$$
\gamma_{2}=-\frac{1}{16 \pi^{4}} \frac{3 c_{F}}{2} N_{f}
$$

We consider now the lattice regularization of QCD given by the Wilson action. Bare operators are defined as local analytic functions of elementary fields, i.e. $U_{\mu}(x)$ and $\psi(x)$. At finite lattice spacing the regularized version of a continuum operator is not unique, indeed regularized versions of the same continuum operator can differ by $O(a)$ terms (at the classical level). We consider the following lattice regularizations of $j_{\mu}^{5}(x), \partial_{\mu} j_{\mu}^{5}(x)$ and $q(x)$ :

$$
\begin{gathered}
a^{3} j_{\mu}^{5, L}=\frac{1}{2}\left[\bar{\psi}(x+\mu) \gamma_{\mu} \gamma_{5} U_{\mu}^{\dagger}(x) \psi(x)+\bar{\psi}(x) \gamma_{\mu} \gamma_{5} U_{\mu}(x) \psi(x+\mu)\right] \\
a \Delta_{\mu} j_{\mu}^{5, L}=\left[j_{\mu}^{5, L}(x)-j_{\mu}^{5, L}(x-\mu)\right]
\end{gathered}
$$

(these definitions come out naturally from the lattice Ward identities associated to an axial vector flavour-singlet transformation [12]), and

$$
a^{4} q^{L}=-\frac{1}{2^{4} \times 32 \pi^{2}} \sum_{\mu \nu \rho \sigma= \pm 1}^{ \pm 4} \epsilon_{\mu \nu \rho \sigma} \operatorname{Tr}\left[\Pi_{\mu \nu} \Pi_{\rho \sigma}\right]
$$

( $\Pi_{\mu \nu}$ is the product of link variables around a plaquette) [13, which has been largely used in quenched lattice QCD studies. The renormalization of $q^{L}$ in pure gauge theory has been studied perturbatively in Ref. [14]; a nonperturbative study for $\mathrm{SU}(3)$ was performed in Ref. [6].

The $r$-term in the Wilson action breaks explicitly chiral invariance, which should be recovered at a non-trivial value of the bare quark masses. Perturbative calculations and general arguments support the existence of a chiral limit, in which the axial anomaly should be reproduced [12, 15, 16]. In that limit, any mixings of $\Delta_{\mu} j_{\mu}^{5, L}$ and $q^{L}$ with lower dimension operators, in particular $\bar{\psi} \gamma_{5} \psi$, must necessarily vanish, for the anomaly equation to hold. Indeed, mixing with $\bar{\psi} \gamma_{5} \psi$ should be completely absorbed in a redefinition of renormalized quark masses; thus, in the chiral limit (i.e. $m_{\pi} \rightarrow 0$ ), only the operators $\Delta_{\mu} j_{\mu}^{5, L}$ and $q^{L}$ need be considered in a renormalization analysis. In the following we study the renormalization of $\Delta_{\mu} j_{\mu}^{5, L}$ and $q^{L}$ in the chiral limit. A more general analysis for non-zero renormalized mass values (i.e. $m_{\pi} \neq 0$ ) requires the introduction of a regularized version of $m \bar{\psi} \gamma_{5} \psi$, but this should not lead to more than few straightforward changes in the following considerations.

Since we are interested in the matrix elements of operators in the $\overline{\mathrm{MS}}$ scheme, we must renormalize our bare lattice operators $j_{\mu}^{5, L}$ and $q^{L}$ in such a way that their renormalized correlation functions coincide with those obtained in $\overline{\mathrm{MS}}$. This should be achieved by a lattice renormalization matrix $Z_{L}$ :

$$
\left(\begin{array}{c}
i 2 N_{f} q \\
\partial_{\mu} j_{\mu}^{5}
\end{array}\right)^{R}=\left(\begin{array}{cc}
Z_{q q} & Z_{q \psi} \\
Z_{\psi q} & Z_{\psi \psi}
\end{array}\right)\left(\begin{array}{c}
i 2 N_{f} q^{L} \\
\Delta_{\mu} j_{\mu}^{5, L}
\end{array}\right)
$$

It is worth mentioning at this point that, unlike the case of flavor-nonsinglet currents, a nonperturbative evaluation of the matrix elements of $Z_{L}$ cannot be obtained by use of Ward identities according to the method developed in Ref. [15]. 
Since the anomalous dimension matrix $\gamma_{\overline{\mathrm{MS}}}(g)$ must depend only on the renormalization scheme, and not on the type of regularization, we must have

$$
\mu \frac{\mathrm{d} Z_{L}}{\mathrm{~d} \mu} Z_{L}^{-1}=\gamma_{\overline{\mathrm{MS}}}(g)
$$

This provides some constraints on the form of $Z_{L}$, in particular on its logarithms. We should have that: $(i) Z_{q q}$ is a finite function of the bare lattice coupling $g_{0} ;(i i)$ The logarithms appear at order $g_{0}^{4}$ in $Z_{q \psi}$ and $Z_{\psi \psi} ;($ iii $) Z_{\psi q}=0$ [16]. Therefore

$$
\begin{aligned}
& Z_{q q}=1+g_{0}^{2} d_{q q}^{(1)}+g_{0}^{4} d_{q q}^{(2)}+O\left(g_{0}^{6}\right) \\
& Z_{q \psi}=g_{0}^{4}\left(c_{q \psi}^{(2)} \ln a \mu+d_{q \psi}^{(2)}\right)+O\left(g_{0}^{6}\right) \\
& Z_{\psi \psi}=1+g_{0}^{2} d_{\psi \psi}^{(1)}+g_{0}^{4}\left(c_{\psi \psi}^{(2)} \ln a \mu+d_{\psi \psi}^{(2)}\right)+O\left(g_{0}^{6}\right) .
\end{aligned}
$$

Some of the above coefficients are known. It is easy to see that $d_{q q}^{(1)}$ is equal to the corresponding coefficient of the multiplicative renormalization of $q^{L}$ in a pure gauge theory [14.17.

$$
d_{q q}^{(1)}=N\left[-\frac{1}{4 N^{2}}+Z_{0}+\frac{1}{8}+\frac{1}{2 \pi^{2}}\right]
$$

where $Z_{0}=0.15493 . d_{q q}^{(1)} \simeq 0.9084$ for $N=3$. From Eq. (15)

$$
c \equiv c_{q \psi}^{(2)}=c_{\psi \psi}^{(2)}=-\frac{1}{16 \pi^{4}} \frac{3 c_{F}}{8} N_{f} .
$$

$d_{\psi \psi}^{(1)}$ depends on the Wilson parameter $r$; for $r=1$, we find, using the results of Ref. [18, $d_{\psi \psi}^{(1)} \simeq-0.073$.

In the following we set

$$
O_{R}(\mu)=\left(\begin{array}{c}
i 2 N_{f} q \\
\partial_{\mu} j_{\mu}^{5}
\end{array}\right)^{R}, \quad O_{L}=\left(\begin{array}{c}
i 2 N_{f} q^{L} \\
\Delta_{\mu} j_{\mu}^{5, L}
\end{array}\right)
$$

Under a change of scale, the matrix elements of $O_{R}(\mu)$ satisfy

$$
\mu \frac{\mathrm{d}}{\mathrm{d} \mu}\left\langle O_{R}\right\rangle=\gamma_{\overline{\mathrm{MS}}}\left\langle O_{R}\right\rangle
$$

whose formal solution is

$$
\left\langle O_{R}\right\rangle\left(\mu^{\prime}\right)=\left[\mathcal{P} \exp \int_{g(\mu)}^{g\left(\mu^{\prime}\right)} \frac{\gamma_{\overline{\mathrm{MS}}}(\tilde{g})}{\beta_{\overline{\mathrm{MS}}}(\tilde{g})} \mathrm{d} \tilde{g}\right]\left\langle O_{R}\right\rangle(\mu)
$$

where $\beta_{\overline{\mathrm{MS}}}(g)$ is the $\beta$-function in the $\overline{\mathrm{MS}}$ scheme and the symbol $\mathcal{P}$ indicates path ordering. Given the particular form of the matrix $\gamma_{\overline{\mathrm{MS}}}$ in our case, it is easy to see that $\mathcal{P}$ may be dropped. Proceeding as in Ref. [19], we choose $\mu^{\prime}=1 / a$ and write

$$
\left\langle O_{R}\right\rangle(1 / a)=Z_{L}(1 / a)\left\langle O_{L}\right\rangle=\left[\exp \int_{0}^{g(1 / a)} \frac{\gamma_{\overline{\mathrm{MS}}}(\tilde{g})}{\beta_{\overline{\mathrm{MS}}}(\tilde{g})} \mathrm{d} \tilde{g}\right]\left[\exp \int_{g(\mu)}^{0} \frac{\gamma_{\overline{\mathrm{MS}}}(\tilde{g})}{\beta_{\overline{\mathrm{MS}}}(\tilde{g})} \mathrm{d} \tilde{g}\right]\left\langle O_{R}\right\rangle(\mu)
$$


and therefore

$$
\left\langle O_{L}\right\rangle=\left[Z_{L}(1 / a)^{-1} \exp \int_{0}^{g(1 / a)} \frac{\gamma_{\overline{\mathrm{MS}}}(\tilde{g})}{\beta_{\overline{\mathrm{MS}}}(\tilde{g})} \mathrm{d} \tilde{g}\right]\left[\exp \int_{g(\mu)}^{0} \frac{\gamma_{\overline{\mathrm{MS}}}(\tilde{g})}{\beta_{\overline{\mathrm{MS}}}(\tilde{g})} \mathrm{d} \tilde{g}\left\langle O_{R}\right\rangle(\mu)\right]
$$

$g(\mu)=Z_{g}^{L}(\mu) g_{0}$, where $g(\mu)$ is the coupling renormalized in $\overline{\mathrm{MS}}$ [20,21].

Notice that the vector

$$
M(\mu)\left\langle O_{R}\right\rangle(\mu) \equiv \exp \int_{g(\mu)}^{0} \frac{\gamma_{\overline{\mathrm{MS}}}(\tilde{g})}{\beta_{\overline{\mathrm{MS}}}(\tilde{g})} \mathrm{d} \tilde{g}\left\langle O_{R}\right\rangle(\mu)
$$

is renormalization group invariant, that is independent of $\mu$, and it is what can be naturally extracted also from physical experiments. The lattice dependence is restricted to

$$
L\left(g_{0}\right) \equiv Z_{L}(1 / a)^{-1} \exp \int_{0}^{g(1 / a)} \frac{\gamma_{\overline{\mathrm{MS}}}(\tilde{g})}{\beta_{\overline{\mathrm{MS}}}(\tilde{g})} \mathrm{d} \tilde{g}
$$

The matrix $M(\mu)$ can be expanded in powers of $g(\mu)$ :

$$
M(\mu)=\left(\begin{array}{cc}
1 & \frac{c}{2 b_{0}} g(\mu)^{2} \\
0 & 1+\frac{c}{2 b_{0}} g(\mu)^{2}
\end{array}\right)+O\left(g(\mu)^{4}\right)
$$

where $b_{0}$ is the first coefficient of the $\beta$-function:

$$
b_{0}=\frac{1}{16 \pi^{2}}\left(\frac{11}{3} N-\frac{2}{3} N_{f}\right) .
$$

Notice that

$$
\frac{c}{2 b_{0}}=-\frac{N_{f}}{\pi^{2}}\left(\frac{11}{3} N-\frac{2}{3} N_{f}\right)^{-1}
$$

is a small number: $\simeq-0.0486$ for $N=3$ and $N_{f}=4$.

$L\left(g_{0}\right)$ can be expanded in powers of $g_{0}$; to order $g_{0}^{2}$ :

$$
L\left(g_{0}\right)=\left(\begin{array}{cc}
1-d_{q q}^{(1)} g_{0}^{2} & -\frac{c}{2 b_{0}} g_{0}^{2} \\
0 & 1-\left(d_{\psi \psi}^{(1)}+\frac{c}{2 b_{0}}\right) g_{0}^{2}
\end{array}\right)+O\left(g_{0}^{4}\right) .
$$

Use of the anomaly equation for the renormalized operators simplifies considerably the above formulae; indeed, in the chiral limit,

$$
\partial_{\mu} j_{\mu}^{5}(x)^{R}=i 2 N_{f} q(x)^{R}
$$

implying

$$
M(\mu)\left\langle O_{R}\right\rangle(\mu)=\left(\begin{array}{c}
\langle T\rangle \\
\langle T\rangle
\end{array}\right)
$$

where 


$$
\langle T\rangle \equiv\left\langle\partial_{\mu} j_{\mu}^{5}(x)^{R}\right\rangle \exp \int_{g(\mu)}^{0} \frac{\bar{\gamma}(\tilde{g})}{\beta_{\overline{\mathrm{MS}}}(\tilde{g})} \mathrm{d} \tilde{g}
$$

We recall that $\bar{\gamma}(g)$ has been defined in Eq. (8). Thus, measuring the matrix element $\left\langle q^{L}\right\rangle$ by Monte Carlo simulation, we obtain

$$
\left\langle i 2 N_{f} q^{L}\right\rangle=z_{q}\left(g_{0}^{2}\right)\langle T\rangle
$$

where

$$
z_{q}\left(g_{0}^{2}\right)=L_{11}+L_{12}
$$

Notice that $\langle T\rangle$ is renormalization group invariant.

From Eq. (29) we find $z_{q}\left(g_{0}^{2}\right)=1-0.8598 g_{0}^{2}+O\left(g_{0}^{4}\right)$ for $N=3$ and $N_{f}=4$. We recall that the typical values of $g_{0}^{2}$ where simulations on today's supercomputer can be performed are actually not small: $g_{0}^{2} \simeq 1$. Therefore as a consequence of the large $O\left(g_{0}^{2}\right)$ coefficient, more terms turn out to be necessary to obtain an estimate of $z_{q}\left(g_{0}^{2} \simeq 1\right)$ from perturbation theory. Of course an improved lattice topological charge density operator having a smaller $O\left(g_{0}^{2}\right)$ term in $z_{q}\left(g_{0}^{2}\right)$ would be welcome. The evaluation of the $O\left(g_{0}^{4}\right)$ term in $z_{q}\left(g_{0}^{2}\right)$ needs the calculation of the coefficients $d_{q q}^{(2)}$ and $d_{q \psi}^{(2)}$ in Eqs. (16) and $\gamma_{3}$ in Eq. (9). $d_{q q}^{(2)}$ requires a two loop lattice calculation. However an estimate may be obtained from the nonperturbative determination of the multiplicative renormalization of $q^{L}$ in quenched QCD, performed in Ref. [6], supplemented with relatively easier analytical two loop calculations of the only graphs containing fermion lines among those contributing to the two-point gluonic function with an insertion of $q^{L}$. The evaluation of the other coefficients should be simpler, $d_{q \psi}$ can be obtained from a one loop calculation, while $\gamma_{3}$ requires a two loop calculation in dimensional regularization.

Given the difficulties of a perturbative evaluation, a nonperturbative estimate of $z_{q}\left(g_{0}^{2}\right)$ would be welcome. To this purpose, notice that the result (33) is independent of the particular matrix element. We may therefore eliminate the lattice factor $z_{q}\left(g_{0}^{2}\right)$, masking the physical signal, by measuring two different matrix elements of $q_{L}$. For example, beside the on-shell nucleon matrix element, we may also extract the matrix element $\left\langle 0\left|q^{L}\right| \eta^{\prime}\right\rangle$ from a lattice calculation. The wall-wall correlation function $G_{q}^{\mathrm{w}}(t)$ constructed from $\left\langle q^{L}(x) q^{L}(y)\right\rangle$ should have the following long distance behavior

$$
G_{q}^{\mathrm{w}}(t) \simeq \frac{\left|\left\langle 0\left|q^{L}\right| \eta^{\prime}\right\rangle\right|^{2}}{2 m_{\eta^{\prime}}} e^{-m_{\eta^{\prime}} t}
$$

Furthermore

$$
\left\langle 0\left|i 2 N_{f} q^{L}\right| \eta^{\prime}\right\rangle=z_{q}\left\langle 0|T| \eta^{\prime}\right\rangle
$$

and

$$
\left\langle 0|T| \eta^{\prime}\right\rangle=\sqrt{N_{f}} m_{\eta^{\prime}}^{2} f_{\eta^{\prime}} .
$$

In the large- $N$ limit $f_{\eta^{\prime}}=f_{\pi}$ [22]. A more precise phenomenological estimate gives $f_{\eta^{\prime}} \simeq$ $0.8 f_{\pi}$ 23.24. 
We have thus seen how a Monte Carlo measurement of the proton matrix element of $q^{L}$, in the presence of dynamical fermions, can be related to the physical, renormalization group invariant quantity relevant for the spin crisis problem; the renormalization function involved in this process can be determined nonperturbatively from measurements of $\left\langle q^{L}(x) q^{L}(y)\right\rangle$ and an estimate of $f_{\eta^{\prime}}$.

Acknowledgements We would like to thank G. Paffuti for helpful conversations. 


\section{REFERENCES}

[1] R. D. Carlitz, Proceedings, XXVI Int. Conf. on High Energy Physics, Dallas (1992), ed. J. R. Sanford, and references therein.

[2] J. Ellis and M. Karliner, Phys. Lett. B313 (1993) 131, and references therein.

[3] G. Veneziano, Mod. Phys. Lett. A4 (1989) 1605; G.M. Shore and G. Veneziano, Mod. Phys. Lett. A8 (1993) 373.

[4] R. Gupta and J. E. Mandula, Phys. Rev. D 50 (1994) 6931.

[5] J. E. Mandula, Phys. Rev. Lett. 65 (1990) 1403; Nucl. Phys. B (Proc. Suppl.) 26 (1992) 356.

[6] B. Allés, M. Campostrini, L. Del Debbio, A. Di Giacomo, H. Panagopoulos and E. Vicari, Phys. Lett B336 (1994) 248.

[7] R. Altmeyer, M. Göckeler, R. Horsley, E. Laermann, G. Schierholz, Nucl. Phys. B (Proc. Suppl.) 30 (1993) 483.

[8] M. Fukugita, Y. Kuramashi, M. Osaka, and A. Ukawa, KEK preprint 94-173, hep-lat 9501010 (1994).

[9] S.J. Dong and K.F. Liu, preprint UK/94-07, hep-lat 9412059 (1994).

[10] D. Espriu and R. Tarrach, Zeitschr. f. Physik C16 (1982) 77.

[11] G.M. Shore and G. Veneziano, Nucl. Phys. B381 (1992) 23.

[12] L.H. Karsten and J. Smit, Nucl. Phys. B183 (1981) 103.

[13] P. Di Vecchia, K. Fabricius, G.C. Rossi and G. Veneziano, Nucl. Phys. B192 (1981) 392.

[14] M. Campostrini, A. Di Giacomo and H. Panagopoulos, Phys. Lett. B212 (1988) 206.

[15] M. Bochicchio, L. Maiani, G. Martinelli and M. Testa, Nucl. Phys. B262 (1985) 331.

[16] J. Smit and J.C. Vink, Nucl. Phys. B284 (1987) 234.

[17] B. Allés and E. Vicari, Phys. Lett. B276 (1992) 148.

[18] G. Martinelli and Z. Yi-Cheng, Phys. Lett. B125 (1983) 77.

[19] A. Di Giacomo, H. Panagopoulos and E. Vicari, Phys. Lett. B240 (1990) 423.

[20] A. Hasenfratz and P. Hasenfratz, Phys. Lett. B93 (1980) 165.

[21] H. Kawai, R. Nakayama and K. Seo, Nucl. Phys. B189 (1981) 40.

[22] E. Witten, Nucl. Phys. B156 (1979) 269.

[23] J.F. Donoghue, B.R. Holstein and Y.C.R. Lin, Phys. Rev. Lett. 55 (1985) 2766; J. Bijnens, A. Bramon and F. Cornet, Phys. Rev. Lett. 61 (1988) 1453; J. Bijnens, Int. J. Mod. Phys. A8 (1993) 3045.

[24] H. Aihara et al., Phys. Rev. Lett. 64 (1990) 172. 